\title{
Mitochondrial Hormone Receptors - an Emerging Field of Signaling in the Cell's Powerhouse
}

\author{
Rüdiger Hardeland* \\ University of Goettingen, Germany
}

Received: September 06, 2017; Published: November 09, 2017

*Corresponding author: Rüdiger Hardeland, Johann Friedrich Blumenbach Institute of Zoology and Anthropology, University of Goettingen, Buergerstr. 50, D-37073 Göttingen, Germany

\begin{abstract}
Hormone receptors that are classically located in either cytosol or nucleus or in the plasma membrane are also found in mitochondria. Notably, they belong to different categories, such as proteins mainly known as hormone-dependent transcription factors, receptor tyrosine kinases, multimeric ligand-gated ion channels, and G protein-coupled receptors. Some of them represent mitochondrial variants, whereas others seem to be almost or fully identical with the extra mitochondrial forms. In some cases, mitochondrial receptors are associated with the outer membrane, whereas others are integrated in the inner membrane and act by signaling towards the matrix. In functional terms, some steroid receptors display genomic actions at the mitochondrial chromosome, whereas membrane-bound receptors transmit metabolic effects in the matrix or at the electron transport chain and modulate mitochondrial structure and length or apoptosis.
\end{abstract}

Keywords: Cannabinoid receptor; EGFR; Melatonin receptor; Mitochondria; Multimeric receptors; Steroid receptors

Abbreviations: GPCRs : G Protein Coupled Receptors; GUCY2s : Guanylyl Cyclases; RTKs: Receptor Tyrosine kinases; GR: Glucocorticoid Receptor; GRE: GR Response Element; MnSOD: Mitochondrial Superoxide Dismutase; ETC: Electron Transport Chain; PR: Progesterone Receptor; EGFR: Epidermal Growth Factor Receptor; PKA : Protein kinase A

\section{Introduction}

The classic view of hormone receptors comprises categories of

(i) Membrane-bound proteins, such as G protein-coupled receptors (GPCRs), receptor guanylyl cyclases (GUCY2s) and receptor tyrosine kinases (RTKs), and

(ii) Intracellular, typically genomically acting receptors that exert their effects as transcription factors in the nucleus. In the latter category, the ligands are small, sufficiently lipophilic molecules able to cross the plasma membrane. Some of their receptors associate with their ligands in the cytoplasm, others directly in nucleus, but sometimes, variants of these receptors may be membrane-associated and transmit nongenomic actions, as found, e.g., in some steroid receptors. In recent years, evidence has accumulated that receptors for hormones and other endocrine factors also exist in mitochondria, as will be outlined in this article. Depending on the respective molecules, the knowledge on transfer of the receptors or receptor variants to the mitochondria as well as on the intramitochondrial signaling mechanisms is different. Nevertheless, the details already known to date are fundamentally expanding our understanding of mitochondrial regulation by hormones, and this emerging field provides exciting insights into the participation of mitochondria in the control of cellular functioning.

\section{Steroid, Tyrosine hormone, and Vitamin $\mathrm{D}_{3}$ Receptors in Mitochondria}

In addition to the well-understood genomic actions in the nucleus and several non genomic extra mitochondrial effects, the localization of steroid receptors in mitochondria has been repeatedly described. The presence of a glucocorticoid receptor (GR) in mitochondria had been demonstrated as early as in 1993 [1]. In another pioneering study, which was, however, poorly considered for several years, a GR response element (GRE) was shown to be present in a D-loop of the mitochondrial chromosome [2]. Meanwhile, several investigations have demonstrated GR translocation to mitochondria [1-5], a process that depends on glucocorticoid availability [1-5] and on a mechanism reminiscent of the transfer into the nucleus, also involving heat shock proteins and an immunophilin [4].

Mitochondrial GR localization in conjunction with the existence of a GRE in the mitochondrial chromosome strongly indicates a regulation of mitochondrial gene transcription by glucocorticoids. In addition, the mitochondrial GR seems to be involved in apoptosis 
induction in thymocytes, which may not be surprising with regard to the presence of the proapoptotic machinery in this organelle [5]. Mitochondrial localization has been multiply shown for the estrogen receptor- $\beta$ (ER $\beta$ ) [6,7], the ER variant anyway known for its nongenomic signaling. In addition to modulation of bioenergetics [6], effects on mitochondrial gene expression were assumed [7]. Moreover, translocation of the usually genomically acting ER $\alpha$ was reported [8]. A more specific and, to a certain degree unexpected, nongenomic action has been recently described for this other estrogen receptor.

In breast cancer cells, ER $\alpha$ was shown to physically interact with the mitochondrial superoxide dismutase (MnSOD) [8]. As MnSOD is activated by the mitochondrially localized sirtuin SIRT3 by deacetylation at $\mathrm{K} 68$, an interference with the acetylation status of K68 was studied. These experiments revealed a correlation of $\mathrm{ER} \alpha / \mathrm{MnSOD}$ association with acetylation of this regulatory lysine [8]. Another recent finding of high actuality concerns the role of long noncoding RNAs (IncRNAs) in mitochondrial ER actions [9], results that may find parallels in the regulation of other mitochondrial steroid receptors. The nuclear-encoded lncRNA SRA (steroid receptor RNA activator) is also found in mitochondria. A relationship to ERs exists insofar as estrogens induce the nuclear $S R A$ repressor protein SHARP (SMRT/HDAC1-associated repressor protein).

Another inhibitory action on SRA is caused by the protein SLIRP (SRA stem-loop interacting RNA-binding protein), a player that is present in mitochondria and also modulates mitochondrially encoded RNAs (mtRNAs) and has effects on the electron transport chain (ETC) [9]. These findings indicate that mitochondrially located ERs may be stabilized by SRA, but seem to be inhibited by estrogen-dependent up regulation of SHARP in the nucleus and by actions of SLIRP in the mitochondria. These results and their interpretation strongly underline the importance of the nuclearmitochondrial interplay as well as the previously unexpected complexity of the coordinated relationship between nuclear and mitochondrial genomes.

A mitochondrial progesterone receptor (PR) has been demonstrated and discussed in terms of nongenomic effects [1013]. These actions concern respiratory activity, such as increased mitochondrial membrane potential and oxygen consumption [10-12], a role that would be in line with metabolism-enhancing properties of property of progesterone, as known from the postovulatory increase in temperature. The mitochondrial PR variant has been shown to be truncated, in which N-terminal domains as well as the DNA-binding domain are absent [10]. Therefore, this PR variant can only act nongenomically. Genomic effects in mitochondria via PR would only be possible, if additional variants can be detected. Two variants of the receptor for another intracellularly acting hormone, triiodothyronine (T3), were discovered in mitochondria [14-16]. Both are truncated forms of the receptor TR $\alpha 1$ of different lengths, p43 and p28. The p43 protein was shown to stimulate mitochondrial gene expression and to influence cell differentiation and apoptosis [16], whereas the role of p28 remains to be identified. The vitamin $\mathrm{D}_{3}$ receptor (VDR) was also detected in mitochondria [17-21]. VDR translocation was reported to take place via the permeability transition pore [18]. VDR signaling seems to mainly result in the suppression of respiratory activity [19-21]. Additional effects on lipid metabolism have been discussed.

\section{EGFR, a Receptor Tyrosine Kinase, in Mitochondria}

EGFR (epidermal growth factor receptor) is an example for a receptor tyrosine kinase that has been shown to be translocated to mitochondria [22]. This finding differs from the previously discussed cases insofar as the ligand is not a low-molecular weight molecule such as steroids, T3 and the vitamin $\mathrm{D}_{3}$ hormone, but represents a peptide of 53 amino acids. However, as proteins like the receptors are translocated, there should be no fundamental problem to also translocate the ligand. Mitochondrial EGFR has been studied in detail in non-small-cell lung cancer cells. It was shown to be internalized by endocytosis and, thereafter, attached to mitochondria [22]. The translocation was stimulated by EGF.

EGFR signaling caused several effects concerning the ETC, in particular, increased ATP formation, but also induced changes in mitochondrial structure and distribution, with consequences to enhanced cell motility. By interfering with the mitochondrial fusion factor Mfn1, it shifted the fusion/fission balance towards fission [22]. Notably, the inhibition of mitochondrial fusion leads to facilitated redistribution of the smaller mitochondria within cells, which would be impossible with the longer organelles that can, in the extreme, fuse to large networks. An important advantage of redistribution is avoidance of peripheral mitochondrial depletion.

\section{Mitochondrial Localization of Tetrameric and Pentameric Membrane-bound Receptors}

Translocation of membrane-bound receptors composed of several subunits, frequently heteromers, to mitochondria appears, at first glance, to be rather unlikely. Nevertheless, this has been shown or concluded to be possible. With regard to an endocytosis mechanism that initiates translocation, this is, however, not at all implausible. A report concerning a mitochondrial NMDA receptor, which represents a tetrameric ionotropic glutamate receptor, indicated several actions concerning enhanced production of reactive oxygen species, however, along with reduced cytochrome c release [23], effects that would require in-depth analysis and confirmation.

In liver and brain, several nicotinic acetylcholine receptors were reported to be mitochondrially localized [24-26]. In these tissues, the heteropentameric subtypes $\alpha 7 \beta 2, \alpha 4 \beta 2$ and, to a minor extent, $\alpha 3 \beta 2$ were detected, whereas in the lung, the $\alpha 3 \beta 4$ receptor subtype prevailed [24]. The nicotinic receptors were reported to be associated with the outer mitochondrial membrane and to be involved in the regulation of apoptosis, partially in a protective way. The $5-\mathrm{HT}_{3}$ receptor represents another pentameric, ionotropic receptor type that was found in cardiac mitochondria [27]. However, the receptor was not analyzed with regard to the various 5 - $\mathrm{HT}_{3}$ subtypes, which differ in their composition of subunits. The receptor was reported to increase the respiration control ratio and to inhibit the opening of the permeability transition pore. 


\section{GPCRs in Mitochondria}

Similar to other membrane-bound receptors, GPCRs have to be integrated into membranes. In the case of GPCRs, insertion of seven transmembrane domains is required. Nevertheless, even these larger integral membrane proteins can be translocated to mitochondria. One example concerns the serotonin receptor $5-\mathrm{HT}_{4}$ [27], which differs from the afore-mentioned ionotropic $5-\mathrm{HT}_{3}$. In functional terms, the mitochondrial $5-\mathrm{HT}_{4}$ receptor was reported to decrease the respiration control ratio, contrary to 5 - $\mathrm{HT}_{3}$, whereas the opening of the permeability transition pore was, again, found to be inhibited [27]. Particular insights were obtained by studies on the type- 1 cannabinoid receptor $\mathrm{CB}_{1}$, which is also translocated to mitochondria [28-31].

Apart from its neurobiological relevance, the particular significance of one of these studies [31] concerns the signaling mechanism of the mitochondrially located $\mathrm{CB}_{1}$. It was shown to modulate the activity of the soluble adenylyl cyclase (SAC) in the matrix via the $\alpha$-subunit of a $G_{i}$ protein. This allows conclusions on the orientation of the receptor. It has to be located in the inner membrane, with the C-terminus that has to interact with Gi towards the matrix side and the ligand binding pocket towards the intermembrane space. The functional consequences of this signaling mechanism are reduction of cAMP levels in the matrix and lower protein kinase A (PKA) activity. Insofar this antagonizes the activation of SAC by bicarbonate, which serves to adapt the respiratory electron flux to the activity of the citric acid cycle.

Enhanced matrix PKA activity leads to phosphorylation of ETC subunits, especially in Complex 1, and presumably, also in other sites. The up regulation of phosphorylation of Complex 1 subunits as initiated by bicarbonate enhances electron feeding to the ETC, whereas the down regulation via $\mathrm{CB}_{1}$ decreases the entrance of electrons into the ETC. These findings on the effects mitochondrial $\mathrm{CB}_{1}$ were also important for interpreting the mitochondrial action of the melatonin receptor $\mathrm{MT}_{1}$. This receptor subtype was shown to be located in mitochondria, contrary to $\mathrm{MT}_{2}$ [32]. Under basal, non compromised conditions, melatonin regulates respiration and Complex 1 activity in a similar way as cannabinoids via $\mathrm{CB}_{1}$. Therefore, it was concluded that $\mathrm{MT}_{1}$ has to be correspondingly oriented in the inner mitochondrial membrane and acts via $\mathrm{G}_{i}$, sAC inhibition, decrease of cAMP concentration and PKA activity [33].

\section{Conclusion}

The localization of hormone receptors in mitochondria considerably expands our insights into the actions of their ligands. It is a remarkable fact that entirely different types of receptors, which are, from a conventional point of view, either present in the cytosol and/or nucleus or in the plasma membrane, can be found in mitochondria. Moreover, the membrane-bound receptors belong to different categories, such as RTKs, multimeric ligandgated ion channels or GPCRs. This multiplicity sheds light on the complexity of cellular processes which connect nuclear, cytosolic and mitochondrial functions in a concerted way. This emerging field will certainly gain increasing future importance and unravel numerous poorly understood connections in cell biology and biomedicine as well.

\section{References}

1. Demonacos C, Tsawdaroglou NC, Djordjevic-Markovic R, Papalopoulou M, Galanopoulos V, et al. (1993) Import of the glucocorticoid receptor into rat liver mitochondria in vivo and in vitro. J Steroid Biochem Mol Biol 46(3): 401-413.

2. Demonacos C, Djordjevic-Markovic R, Tsawdaroglou N, Sekeris CE (1995) The mitochondrion as a primary site of action of glucocorticoids: the interaction of the glucocorticoid receptor with mitochondrial DNA sequences showing partial similarity to the nuclear glucocorticoid responsive elements. J Steroid Biochem Mol Biol 55(1): 43-55.

3. Psarra AM, Sekeris CE (2011) Glucocorticoids induce mitochondrial gene transcription in HepG2 cells: role of the mitochondrial glucocorticoid receptor. Bio chim Biophys Acta 1813(10): 1814-1821.

4. Nicolaides NC, Charmandari E (2017) Novel insights into the molecular mechanisms underlying generalized glucocorticoid resistance and hypersensitivity syndromes. Hormones (Athens) 16(2): 124-138.

5. Prenek L, Boldizsár F, Kugyelka R, Ugor E, Berta G, et al. (2017) The regulation of the mitochondrial apoptotic pathway by glucocorticoid receptor in collaboration with Bcl-2 family proteins in developing $\mathrm{T}$ cells. Apoptosis 22(2): 239-253.

6. Liao TL, Tzeng CR, Yu CL, Wang YP, Kao SH (2015) Estrogen receptor- $\beta$ in mitochondria: implications for mitochondrial bioenergetics and tumorigenesis. Ann NY Acad Sci 1350: 52-60.

7. Chmielewska M, Skibińska I, Kotwicka M (2017) Mitochondria: Target organelles for estrogen action. Postepy Hig Med Dosw 71(0): 454-465.

8. Lone MU, Baghel KS, Kanchan RK, Shrivastava R, Malik SA, et al. (2017) Physical interaction of estrogen receptor with MnSOD: implication in mitochondrial $\mathrm{O}_{2}(.-)$ upregulation and mTORC2 potentiation in estrogen-responsive breast cancer cells. Oncogene 36(13): 1829-1839.

9. Dong Y, Yoshitomi T, Hu JF, Cui J (2017) Long noncoding RNAs coordinate functions between mitochondria and the nucleus. Epigenetics Chromatin 10(1): 41.

10. Dai Q, Shah AA, Garde RV, Yonish BA, Zhang L, et al. (2013) A truncated progesterone receptor (PR-M) localizes to the mitochondrion and controls cellular respiration. Mol Endocrinol 27(5): 741-753.

11. Feng Q, Crochet JR, Dai Q Leppert PC, Price TM (2014) Expression of a mitochondrial progesterone receptor (PR-M) in leiomyomata and association with increased mitochondrial membrane potential. J Clin Endocrinol Metab 99(3): E390-E399

12. Tantibhedhyangkul J, Hawkins KC, Dai Q, Mu K, Dunn CN, et al. (2014) Expression of a mitochondrial progesterone receptor in human spermatozoa correlates with a progestin-dependent increase in mitochondrial membrane potential. Andrology 2(6): 875-883.

13. Price TM, Dai Q (2015) The role of a mitochondrial progesterone receptor (PR-M) in progesterone action. Semin Reprod Med 33(3): 185194.

14. Bertrand Gaday C, Pessemesse L, Cabello G, Wrutniak Cabello C, Casas F (2016) Temperature homeostasis in mice lacking the p43 mitochondrial T3 receptor. FEBS Lett 590(7): 982-991.

15. Cvoro A, Bajic A, Zhang A, Simon M, Golic I, et al. (2016) Ligand independent and subtype-selective actions of thyroid hormone receptors in human adipose derived stem cells. PLoS One 11(10): e016440710.

16. Wrutniak-Cabello C, Casas F, Cabello G (2017) Mitochondrial T3 receptor and targets. Mol Cell Endocrinol. [Epub ahead of print, Feb 3] doi: 10/1016/j.mce.2017.01.054. 
17. Silvagno F, De Vivo E, Attanasio A, Gallo V, Mazzucco G, et al. (2010) Mitochondrial localization of vitamin D receptor in human platelets and differentiated megakaryocytes. PLoS One 5(1): e8670.

18. Silvagno F, Consiglio M, Foglizzo V, Destefanis M, Pescarmona G (2013) Mitochondrial translocation of vitamin $\mathrm{D}$ receptor is mediated by the permeability transition pore in human keratinocyte cell line. PLoS One 8(1): e54716.

19. Consiglio M, Destefanis M, Morena D, Foglizzo V, Forneris M, et al. (2014) the vitamin D receptor inhibits the respiratory chain, contributing to the metabolic switch that is essential for cancer cell proliferation. PLoS One 9(12): e115816.

20. Consiglio M, Viano M, Casarin S, Castagnoli C, Pescarmona G, et al. (2015) Mitochondrial and lipogenic effects of vitamin D on differentiating and proliferating human keratinocytes. Exp Dermatol 24(10): 748-753.

21. Silvagno F, Pescarmona G (2017) Spotlight on vitamin D receptor, lipid metabolism and mitochondria: Some preliminary emerging issues. Mol Cell Endocrinol450: 24-31.

22. Che TF, Lin CW, Wu YY, Chen YJ, Han CL, et al. (2015) mitochondrial translocation of EGFR regulates mitochondria dynamics and promotes metastasis in NSCLC. Oncotarget 6(35): 37349-37366.

23. Korde AS, Maragos WF (2016) direct exposure to N-methyl-d-aspartate alters mitochondrial function. Neurosci Lett 623: 47-51.

24. Lykhmus O, Gergalova G, Koval L, Zhmak M, Komisarenko S, et al. (2014) Mitochondria express several nicotinic acetylcholine receptor subtypes to control various pathways of apoptosis induction. Int J Biochem Cell Biol 53: 246-252.

25. Chernyavsky AI, Shchepotin IB, Galitovkiy V, Grando SA (2015) Mechanisms of tumor-promoting activities of nicotine in lung cancer: synergistic effects of cell membrane and mitochondrial nicotinic acetylcholine receptors. BMC Cancer 15: 152.

26. Chernyavsky A, Chen Y, Wang PH, Grando SA (2015) Pemphigus vulgaris antibodies target the mitochondrial nicotinic acetylcholine receptors that protect keratinocytes from apoptolysis. Int Immunopharmacol 29(1): 76-80.

27. Wang Q Zhang H, Xu H, Guo D, Shi H, et al. (2016) 5-HTR3 and 5-HTR4 located on the mitochondrial membrane and functionally regulated mitochondrial functions. Sci Rep 6: 37336.

28. Bénard G, Massa F, Puente N, Lourenço J, Bellocchio L, et al. (2012) Mitochondrial $\mathrm{CB}_{1}$ receptors regulate neuronal energy metabolism. Nat Neurosci 15(4): 558-564.

29. Hebert Chatelain E, Reguero L, Puente N, Lutz B, Chaouloff F, et al. (2014) Cannabinoid control of brain bioenergetics: Exploring the subcellular localization of the $\mathrm{CB}_{1}$ receptor. Mol Metab 3(4): 495-504.

30.Xu Z, Lv XA, Dai Q Ge YQ Xu J (2016) Acute upregulation of neuronal mitochondrial type-1 cannabinoid receptor and its role in metabolic defects and neuronal apoptosis after TBI. Mol Brain 9(1): 75.

31. Hebert Chatelain E, Desprez T, Serrat R, Bellocchio L, Soria-Gomez E, et al. (2016) a cannabinoid link between mitochondria and memory. Nature 539(7630): 555-559.

32. Wang X, Sirianni A, Pei Z, Cormier K, Smith K, et al. (2011) The melatonin MT1 receptor axis modulates mutant Huntingtin-mediated toxicity. J Neurosci 31: 14496-14507.

33. Hardeland R (2017) Melatonin and the electron transport chain. Cell Mol Life Sci. 74(21): 3883-3896

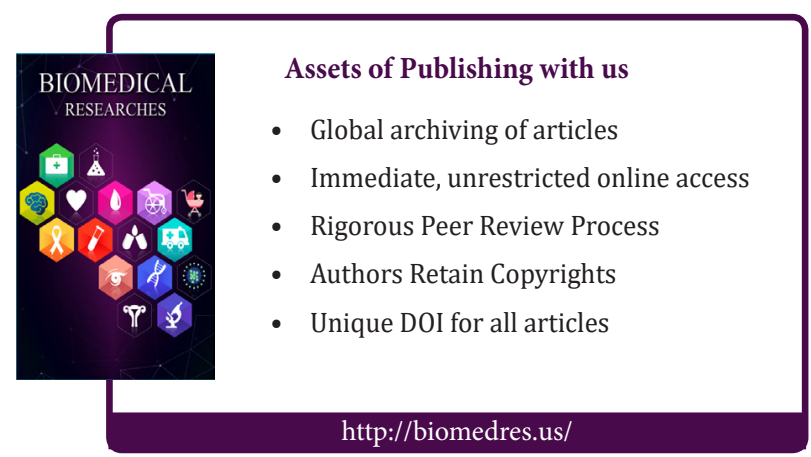

